\title{
CARACTERIZACIÓN QUÍMICA, FÍSICA Y MICROBIOLÓGICA DE DOS VINAZAS CUBANAS
}

\author{
YUSLEIDI PÉREZ OLIVA ${ }^{1}$ \\ OSNEY PEREZ ONES ${ }^{2}$ \\ LOURDES ZUMALACÁRREGUI DE CÁRDENAS ${ }^{3}$
}

\section{RESUMEN}

En la presente investigación se realizó la caracterización química, física y microbiológica de las vinazas resultantes de la producción de etanol y aguardiente a partir de miel final, de dos destilerías cubanas: Héctor Molina y San José. Se determinaron parámetros de importancia para evaluar la calidad de las mismas, como son demanda biológica de oxígeno $\left(\mathrm{DBO}_{5}\right)$, demanda química de oxígeno (DQO), potasio, hierro, coliformes totales y termotolerantes, entre otros. Las destilerías Héctor Molina y San José presentaron similitud en cuanto a: $\mathrm{pH}, \mathrm{DBO}_{5}$ y DQO. La valoración estadística que se realizó arrojó que existen diferencias significativas entre las variables $\mathrm{DBO}_{5}, \mathrm{DQO}, \mathrm{NO}_{3}{ }^{-}, \mathrm{PO}_{4}{ }^{3-}, \mathrm{SO}_{4}{ }^{2-}, \mathrm{Mg}^{2+}, \mathrm{Na}^{+}, \mathrm{K}^{+}$, $\mathrm{Ag}, \mathrm{Ba}, \mathrm{Mn}, \mathrm{Ni}, \mathrm{Cr}$, Ca y sólidos sedimentables. Además se comparó con la Norma Cubana 27:2012, encontrándose que las variables: $\mathrm{DBO}_{5}, \mathrm{DQO}$, conductividad eléctrica, $\mathrm{pH}$ y temperatura no cumplen con los límites máximos permisibles reportados por esta.

PALABRAS CLAVES: Destilerías, Etanol, Vinazas, Caracterización, Calidad, Legislación.

\section{CHEMICAL, PHYSICAL AND MICROBIOLOGICAL CHARACTERIZATION OF TWO CUBAN STILLAGE}

\section{ABSTRACT}

In this research the chemical, physical and microbiological characterizations of vinasse from ethanol and rum production at Hector Molina and San José distilleries were performed. Molasses were used as raw material. Important

1 Empresa Nacional de Análisis y Servicios Técnicos, ENAST, Cuba.

2 Facultad de Ingeniería Química. Instituto Superior Politécnico José Antonio Echeverría, Cujae, Cuba.

3 Facultad de Ingeniería Química. Instituto Superior Politécnico José Antonio Echeverría, Cujae, Cuba.

Autor de correspondencia: Pérez Ones, O. (Osney): Instituto Superior Politécnico José Antonio Echeverría, Cujae: Calle 114 No. 11901 e/ Ciclovía y Rotonda, Apartado 6028. Marianao 15, CP 19390, La Habana, Cuba. Tel.: (53) 72663350.

Correo electrónico: osney@quimica.cujae.edu.cu
Historia del artículo:

Artículo recibido: 02-VI-2016 / Aprobado: 18-V-2017

Disponible online: 30 de agosto de 2017

Discusión abierta hasta octubre de 2018 
parameters were determined for the evaluation of their qualities. Some of them were: Biological oxygen demand $\left(\mathrm{BOD}_{5}\right)$, chemical oxygen demand (COD), potassium, iron, total coliforms and thermotolerant coliforms. Distilleries Hector Molina and San José presented similarity, comparing pH, (BOD5) and COD. Statistical evaluation was performed showing that there are significant differences between both distilleries for the variables: $\mathrm{BOD}_{5}, \mathrm{COD}, \mathrm{NO}^{3-}, \mathrm{PO}_{4}^{3-}, \mathrm{SO}_{4}^{2-}, \mathrm{Mg}^{2+}, \mathrm{Na}^{+}, \mathrm{K}+$, $\mathrm{Ag}, \mathrm{Ba}, \mathrm{Mn}, \mathrm{Ni}, \mathrm{Cr}$, Ca and settleable solids. Those values were compared with the Cuban Standard 27: 2012, finding that $\left(\mathrm{BOD}_{5}\right), \mathrm{COD}$, electrical conductivity, $\mathrm{pH}$ and temperature do not comply with the maximum permissible levels reported.

KEYWORDS: Breweries, Ethanol, Stillage, Characterization, Quality, Legislation.

\section{CARACTERIZAÇÃo QUÍMICA, FÍSICA E MICROBIOLÓGICA DE DOIS VINHAÇA CUBANAS}

\section{RESUMO}

Nesta pesquisa a caracterização química, física e microbiológica da vinhaça da produção de etanol a partir de melaço e rum, destilarias Hector Molina e San Jose ocorreu. Parâmetros importantes foram determinados para a qualidade deles, como demanda biológica de oxigênio (DB05), demanda química de oxigênio (DQ0), potássio, ferro, coliformes totais, termotolerantes, entre outros. Destilarias Hector Molina e San José apresentou similaridade de pH, (DBO5) e COD. A avaliação estatística foi realizada que mostrou que há diferenças significativas entre as variáveis $\mathrm{NO}_{3}{ }^{-}, \mathrm{PO}_{4}{ }^{3}, \mathrm{DQO}$, sólidos sedimentáveis, $\mathrm{Ag}, \mathrm{Ba}, \mathrm{Mg}^{2+}, \mathrm{Na}^{+}, \mathrm{K}^{+}, \mathrm{Mn}, \mathrm{Ni}, \mathrm{Cr}, \mathrm{SO}_{4}{ }^{2-}$, Ca e DBO. Além disso, em comparação com o Internacional Standard 27: 2012, descobrindo que as variáveis: (DBO5), DQO, condutividade elétrica, pH e temperatura não cumpram os níveis máximos admissíveis relatados por isso.

PALAVRAS-CHAVE: Cervejarias, Vinhaça etanol, Caracterização, Qualidade, Legislação.

\section{INTRODUCCIÓN}

La dimensión ambiental de cualquier empresa debe analizarse en un sentido amplio, sistémico y holístico, por lo que se deben considerar todos sus aspectos, tanto naturales (suelo, aire, agua, flora, fauna, ecosistemas, entre otros), como los sociales (influencia del hombre sobre la naturaleza y los efectos adversos que genera esta interacción sobre la salud humana) y los económicos (Perdigón y Obregón, 2005). El cuidado del medio ambiente ha sido de gran importancia para la humanidad, pero no fue solo hasta la década del 90 que comienza a hablarse de desarrollo sostenible. El desarrollo de procesos tecnológicos y químicos, con el fin de obtener productos de gran demanda de la población, ha traído como consecuencia fenómenos negativos como son los provocados por los desechos industriales (Diez, 2008).

Las vinazas producidas en destilerías de etanol se encuentran entre los residuales orgánicos de mayor efecto contaminante sobre la flora y la fauna del planeta (Lezcano y Mora, 2011). La problemática principal radica en que por cada litro de etanol producido a partir de miel final, se obtienen, de manera adicional, 10-15 litros de vinaza como residual, con una demanda química de oxígeno ( $\mathrm{DQO}_{5}$ ) entre 60 y $70 \mathrm{~g} / \mathrm{l}$ y un $\mathrm{pH}$ alrededor de 4 . En muchos lugares esta vinaza se vierte en ríos, lagos, presas y canales sin previo tratamiento, por lo que contaminan las fuentes de aguas superficiales y subterráneas con un fuerte impacto negativo sobre el medio ambiente (Lezcano y Mora, 2011). 
Cuba no se encuentra exenta de este problema. El acelerado desarrollo industrial ha estado vinculado a la adopción de restricciones, cada vez más severas, con relación al vertido de efluentes industriales. Las destilerías tienen que dar solución a los problemas de estos efluentes, para cumplir con la legislación vigente NC 27:2012 (Cómite de Normalización 2012).

En Cuba, la industria azucarera y sus derivados aportan anualmente una contaminación equivalente a lo que aportarían siete millones de habitantes (Perdigón y Obregón, 2005). Una de las provincias de Cuba con más desarrollo industrial es Mayabeque. En esta existen tres destilerías: San José, Héctor Molina y Santa Cruz del Norte. La destilería de San José trabaja 24 horas los siete días de la semana. En esta se obtienen 30000 litros de aguardiente diario y 60000 litros de vinaza, cuyo destino final es su conservación en un contenedor hasta su solicitud por otra empresa, pudiendo pararse la producción por no tener donde contener esta vinaza.

La destilería Héctor Molina trabaja 24 horas los siete días de la semana; produce 50000 litros de etanol diario y 80000 litros de vinaza que se vierten a una zanja que drena directamente al mar sin tratamiento alguno.
Es por ello que se propuso como objetivos de esta investigación la caracterización de las vinazas de las destilerías de Héctor Molina Riaño y San José y su comparación según su calidad con la legislación cubana vigente.

\section{MATERIALES Y MÉTODOS}

\subsection{Caracterización de las vïnazas}

Para el estudio se realizó una visita semanal durante 4 meses a cada destilería en estudio, (Héctor Molina Riaño y San José) tomando una muestra de 3 litros en cada visita. Por lo anterior, el tamaño poblacional de las muestras fue 16. El tamaño de cada muestra, 3 litros es debido a que para todos los análisis se necesitaba un poco más de 2 litros. En cada destilería se midió la temperatura de la vinaza a la salida del proceso de destilación, el pH y la conductividad eléctrica. Una vez extraída la muestra de 3 litros en un frasco estéril, se fijó el oxígeno disuelto añadiéndole a cada muestra $2 \mathrm{ml}$ de solución de sulfato de manganeso y se trasladó al laboratorio dentro de una nevera a una temperatura aproximada de $4^{\circ} \mathrm{C}$ para su posterior análisis. En el laboratorio de la Unidad de Base Empresarial de La Habana perteneciente a la Empresa Nacional de Análisis y Servicios Técnicos (ENAST) se determinaron los parámetros que se muestran en la Tabla 2.1.

TABLA 2.1. DETERMINACIONES ANALÍTICAS SEGÚN SU EQUIPAMIENTO

\begin{tabular}{|c|c|c|}
\hline Parámetro & Unidad de medida & Equipo o medio de medición \\
\hline $\mathrm{SO}_{4}^{2-}, \mathrm{NO}_{3}^{-}, \mathrm{PO}_{4}^{3-}$ & $\mathrm{mg} / \mathrm{l}$ & Espectrofotómetro HACH, DR/2010 \\
\hline $\mathrm{Ca}^{2+}, \mathrm{Mg}^{2+}, \mathrm{DQO}, \mathrm{DBO}_{5}{ }^{20}, \mathrm{O}_{2}$ disuelto & $\mathrm{mg} / \mathrm{l}$ & Bureta \\
\hline $\mathrm{Na}^{+}, \mathrm{K}^{+}$ & $\mathrm{mg} / \mathrm{l}$ & Fotómetro de llama Corning 410 \\
\hline $\mathrm{pH}$ & $\mathrm{U}$ & pH-metro GLP 22, CRISON \\
\hline Conductividad eléctrica & $\mathrm{mS} / \mathrm{cm}$ & Conductímetro Basic 30, CRISON \\
\hline Sólidos sedimentables & $\mathrm{ml} / \mathrm{l}$ & Cono Kirchoff \\
\hline $\begin{array}{l}\text { Coliformes totales, coliformes termotolerantes, } \\
\text { levaduras, hongos filamentosos }\end{array}$ & NMP/100 mg/l & Visual \\
\hline Escherichia.coli, Pseudomonas aeruginosa & NMP/100 mg/l & Lámpara fluorescente UVITEC Cambridge \\
\hline $\mathrm{Ag}, \mathrm{Ba}, \mathrm{Cd}, \mathrm{Co}, \mathrm{Cu}, \mathrm{Fe}, \mathrm{Mn}, \mathrm{Mo}, \mathrm{Ni}, \mathrm{Pb}, \mathrm{V}, \mathrm{Zn}, \mathrm{Cr}$. & $\mathrm{mg} / \mathrm{l}$ & $\begin{array}{c}\text { Espectrómetro de emisión atómica con } \\
\text { plasma inductivamente acoplado (ICP-AES), } \\
\text { THERMO JARRELL, ICAP } 61 \mathrm{E}\end{array}$ \\
\hline
\end{tabular}


Cada muestra se caracterizó desde el punto de vista microbiológico, físico y químico. Primero se determinaron las medias poblacionales de cada parámetro evaluado (Tabla 2.1) para determinar si la distribución correspondía a una distribución normal, partiendo de los resultados de las 16 muestras de cada vinaza. Esta medida de tendencia central se determinó utilizando el programa estadístico Statgraphics Centurión XV.

Para la caracterización microbiológica se determinaron coliformes totales y termotolerantes, Pseudomonas, levaduras, hongos y bacterias, realizando los análisis de las 16 muestras de cada vinaza según las técnicas mencionadas en la Tabla 2.2.

Para la caracterización física se estudiaron los parámetros: $\mathrm{pH}$, temperatura, conductividad eléctrica de las 16 muestras de cada vinaza con las técnicas que se mencionan en la Tabla 2.2.

Para la caracterización química se hicieron las determinaciones: contenido de iones calcio, magnesio, sodio y potasio, DBO, DQO, sólidos sedimentables, nitratos, fosfatos y contenido de metales, como se muestra en la Tabla 2.2.

Los medios de cultivo utilizados para los ensayos microbiológicos fueron: caldo lactosado, caldo bilis verde brillante y caldo EC.

Para conocer la existencia de Pseudomonas aeruginosa y Escherichia coli se utilizaron los medios de cultivo agar asparigina, agar cetrimida y EC-mug.

Se determinó el número más probable (NMP) de organismos presentes en un volumen de muestra usando la técnica de tubos múltiples de fermentación, verificando la presencia del microorganismo de interés con el medio de cultivo específico.

Por último se prepararon placas Petri con un medio enriquecido selectivo de agar maltosa de Sabouraud, con el objetivo de observar si existía crecimiento de hongos filamentosos y levaduras.

Todos los procedimientos anteriores se realizaron en la cabina de flujo laminar para evitar las contaminaciones externas que pudieran dar un fal- so positivo y se incubaron durante 24 y 48 horas a $35-37^{\circ} \mathrm{C}$.

\begin{tabular}{|c|c|}
\hline Parámetro & Técnica empleada \\
\hline $\begin{array}{c}\text { Coliformes totales } \\
\text { y termotolerantes, } \\
\text { bacterias (Escherichia } \\
\text { coli), Pseudomonas } \\
\text { aeruginosa, } \\
\text { temperatura, } \\
\text { conductividad eléctrica, } \\
\text { pH, } \mathrm{Mg}^{2+}, \mathrm{Na}^{+}, \mathrm{K}^{+}, \\
\mathrm{DQO}, \mathrm{DBO}_{5}^{20}, \text { sólidos } \\
\text { sedimentables, } \mathrm{O}_{2}, \mathrm{Ag}, \\
\mathrm{Ba}, \mathrm{Cd}, \mathrm{Co}, \mathrm{Cu}, \mathrm{Fe}, \mathrm{Mn}, \\
\mathrm{Mo}, \mathrm{Ni}, \mathrm{Pb}, \mathrm{V}, \mathrm{Zn}, \mathrm{Cr} \text {. }\end{array}$ & $\begin{array}{c}\text { Standard Methods for the } \\
\text { examination of water and } \\
\text { wastewater. 20th Edition. } \\
1998\end{array}$ \\
\hline $\begin{array}{l}\text { Levaduras, hongos } \\
\text { filamentosos }\end{array}$ & $\begin{array}{c}\text { NC-ISO 7954:2002 } \\
\text { Microbiología de alimentos } \\
\text { de consumo humano y } \\
\text { animal. Guía general para } \\
\text { la enumeración de placa } \\
\text { vertida a } 25^{\circ} \mathrm{C} \text {. }\end{array}$ \\
\hline $\mathrm{NO}_{3}, \mathrm{PO}_{4}{ }^{3-}$ & $\begin{array}{l}\text { 26-LAB-MOP-526: } 2011 \text {. } \\
\text { Dosage des ions } \mathrm{PO}_{4}^{-3}, \\
\mathrm{NO}_{2} \text {, } \mathrm{NO}_{3}^{-} \text {et } \mathrm{NH}_{4}^{+} \text {par la } \\
\text { méthode du flux continu. }\end{array}$ \\
\hline
\end{tabular}

En el laboratorio se prepararon los blancos y los patrones para la determinación de la demanda bioquímica de oxígeno (DBO), ya que para realizar este ensayo se requiere que las muestras no hayan sido filtradas, centrifugadas, ni decoloradas, es decir, muestras sin un tratamiento previo.

Para determinar la demanda química de oxígeno (DQO), se preparó suficiente mezcla oxidante (dicromato de potasio y agente ácido) para adicionar $10 \mathrm{ml}$ a cada muestra, blanco y patrón para que estos asimilasen un proceso de digestión de una hora a $121^{\circ} \mathrm{C}$ y $0,1 \mathrm{MPa}$ en autoclave.

La adición de todos los ácidos que intervienen en las determinaciones se realizó bajo una campana de extracción de gases.

Para los otros ensayos físico-químicos se efectuó una digestión añadiendo ácido nítrico y peróxido de hidrógeno a $85^{\circ} \mathrm{C}$ durante 1,5 horas. Posteriormente se enfrió y se procedió con los análisis. 


\subsection{Comparación de las vinazas se-} gún su calidad

Cada uno de los parámetros característicos de las vinazas, se consideraron variables a estudiar estadísticamente. En primer lugar, se determinó si la distribución normal se ajustaba a los datos medidos, para establecer el análisis estadístico adecuado. Si la distribución era normal se realizó una prueba de hipótesis; en caso contrario, se determinaron las medianas para aplicar W de Mann-Whitney (Wilcoxon), como criterio comparativo.

Se empleó el programa estadístico Statgraphics Centurión XV, para un nivel de confianza del $95 \%$, lo que permitió establecer los factores de mayor significancia sobre la variable respuesta.

Para comparar los resultados obtenidos de cada vinaza se realizó un análisis de correlación con el programa estadístico Statgraphics Centurión XV, entre las variables: $\mathrm{pH}$, temperatura, DBO, DQO y oxígeno disuelto debido a que son estas variables las que tienen una relación directa y pueden influir una sobre la otra (Díaz, 1987).

Luego se realizó la comparación de los parámetros medidos de cada vinaza con la Norma $\mathrm{Cu}$ - bana 27:2012 y se corroboró la necesidad de tratar estos residuos para su empleo adecuado.

\section{RESULTADOS Y DISCUSIÓN}

\subsection{Caracterización de las vinazas de} la destillería de San José

A partir de los resultados obtenidos en los ensayos de laboratorio se desarrolló una caracterización microbiológica, física y química de la vinaza como se muestra en las Figuras 3.1-3.5.

No se apreció la presencia de coliformes totales, coliformes termotolerantes, Escherichia coli y Pseudomonasa eruginosas.

Según los datos mostrados en la Figura 3.1 se establece que la vinaza de la destilería de San José, de acuerdo a su caracterización microbiológica, es muy favorable para el crecimiento de los hongos filamentosos, proliferando hasta valores caracterizados como incontables. Esto se justifica al ser un residual rico en azúcares coincidiendo con los resultados de (Ajello, 2012) y (Madrigal, 2000). Todavía en el residuo, las levaduras se siguen reproduciendo, detectándose en ambas vinazas. De acuerdo a los demás parámetros microbiológicos se evidencia que no existe crecimiento de otros microorganismos.

Figura 3.1. Parámetros microbiológicos. Eje x: Número de muestras y Eje y: Número más probable de microorganismos (NMP)

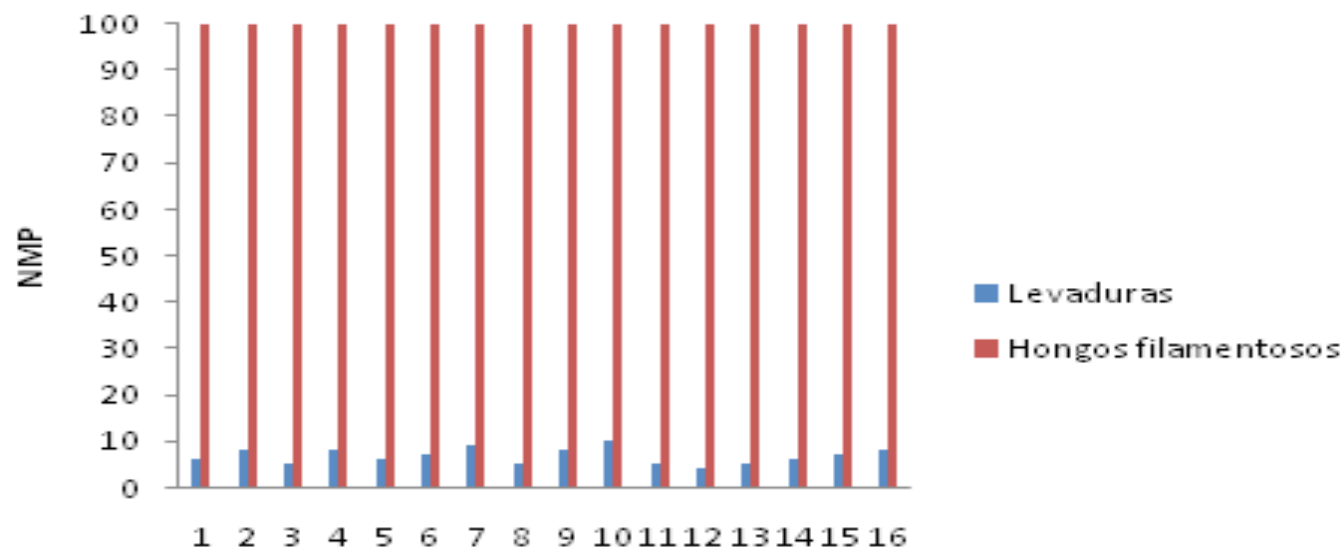

Número de muestras 
Aunque el proceso de producción de aguardiente tiene varios pasos, se puede observar que todavía en el residuo, las levaduras se siguen reproduciendo. Esto se debe a que el medio tiene un $\mathrm{pH}$ ácido, el cual estimula el crecimiento de la mayoría de las levaduras. De acuerdo a los demás parámetros microbiológicos se evidencia que no existe crecimiento de otros microorganismos.

Como se evidenció en la Figura 3.2 se establece que la vinaza de la destilería de San José tiene un $\mathrm{pH}$ ligeramente ácido coincidiendo con los estudios realizados por (Alfaro y Ocampo, 2012). Este comportamiento puede estar asociado a la removilización de metales pesados en suelos, cuyos poros pueden obstruirse por las altas cargas de sólidos en suspensión. Además se propicia el desarrollo de condiciones anaerobias que no solo son perceptibles por sus signos antiestéticos, sino también porque pueden contribuir a la acidificación y a la mencionada removilización de metales pesados. Esto también se debe a la acidificación del mosto con ácido clorhídrico o con ácido sulfúrico.

Estas vinazas poseen un color oscuro debido a la presencia de melanoidinas coincidiendo con los resultados obtenidos por (Ávila, 2013). La temperatura se encuentra en el intervalo $98^{\circ} \mathrm{C}-9^{\circ} 9^{\circ} \mathrm{C}$. La conductividad eléctrica está entre $13 \mathrm{mS} / \mathrm{cm}$ y $15 \mathrm{mS} / \mathrm{cm}$, lo cual indica el poder de salinización que puede generar la vinaza en caso de ser vertida al suelo; similar resultado se obtuvo por (Alfaro y Ocampo, 2012).

\section{Figura 3.2. Parámetros físico-químicos. Eje $\mathrm{x}$ : Número de muestras y Eje y: Concentración (mg/l) y pH}

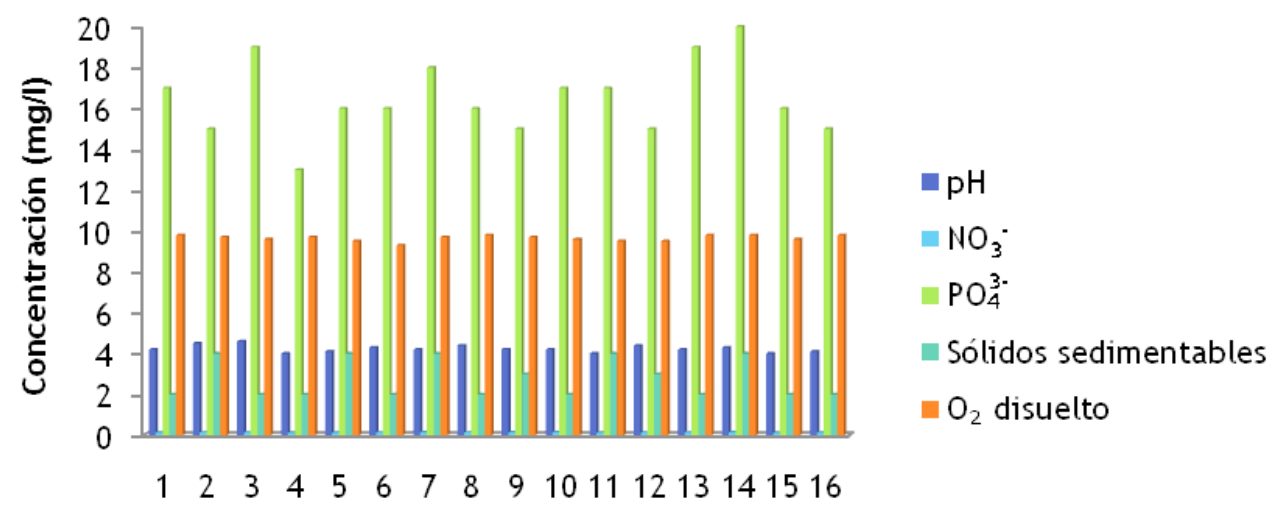

Número de muestras

Figura 3.3. Demanda de oxígeno. Eje x: Número de muestras y Eje y: Concentración (mg/L)

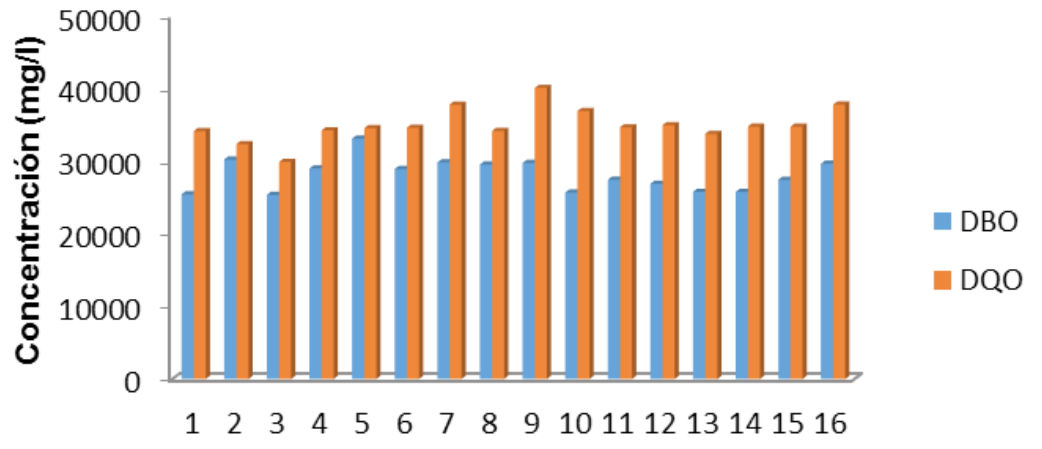

Número de muestras 
La Figura 3.3 muestra los valores de la demanda de oxígeno. En ella se puede observar que la vinaza posee altos valores de DQO y DBO, lo cual coincide con los estudios realizados por (Ávila, 2013), (Alfaro y Ocampo, 2012), (Gandini, 2013) y (Robles y Villalobos, 2011).

La Figura 3.4 demuestra que la vinaza posee altos contenidos de sales, especialmente el contenido de sales de potasio y magnesio. Los resultados coinciden con otros autores que la califican como un residuo rico en potasio y magnesio (Alfaro y Ocampo, 2012), (Gandini, 2013) y (Robles y Villalobos, 2011).

Los otros metales presentan concentraciones menores que $6 \mathrm{mg} / \mathrm{l}$. De aquí que se pueda afirmar que los metales que prevalecen son: hierro y manganeso; generalmente en las vinazas esos son los metales que predominan, por lo que se coincide con los resultados reportados por (Gandini, 2013) y (Robles y Villalobos, 2011).

\subsection{Caracterización de las vinazas} de la destilería Héctor Molina

A partir de los resultados obtenidos se desarrolló una caracterización microbiológica, física y química de las vinazas. Estos resultados se grafican en las Figuras 3.6 - 3.10.

No se apreció la presencia de coliformes totales, coliformes termotolerantes, Escherichia coli y Pseudomonas aeruginosas.

\section{Figura 3.4. Parámetros químicos. Eje x: Número de muestras y Eje y: Concentración (mg/l)}

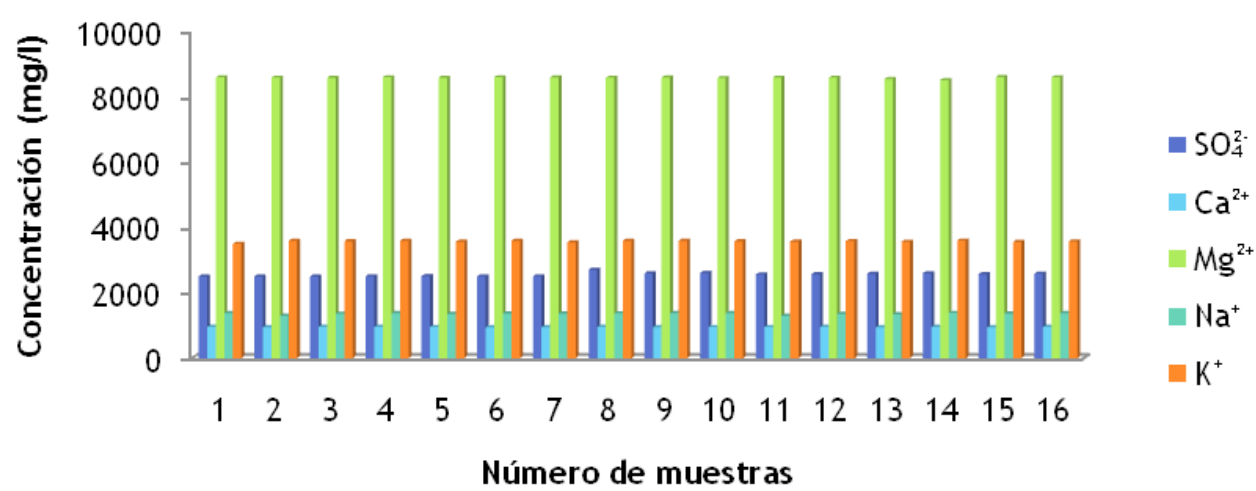

Figura 3.5. Metales. Eje x: Número de muestras y Eje y: Concentración (mg/l)

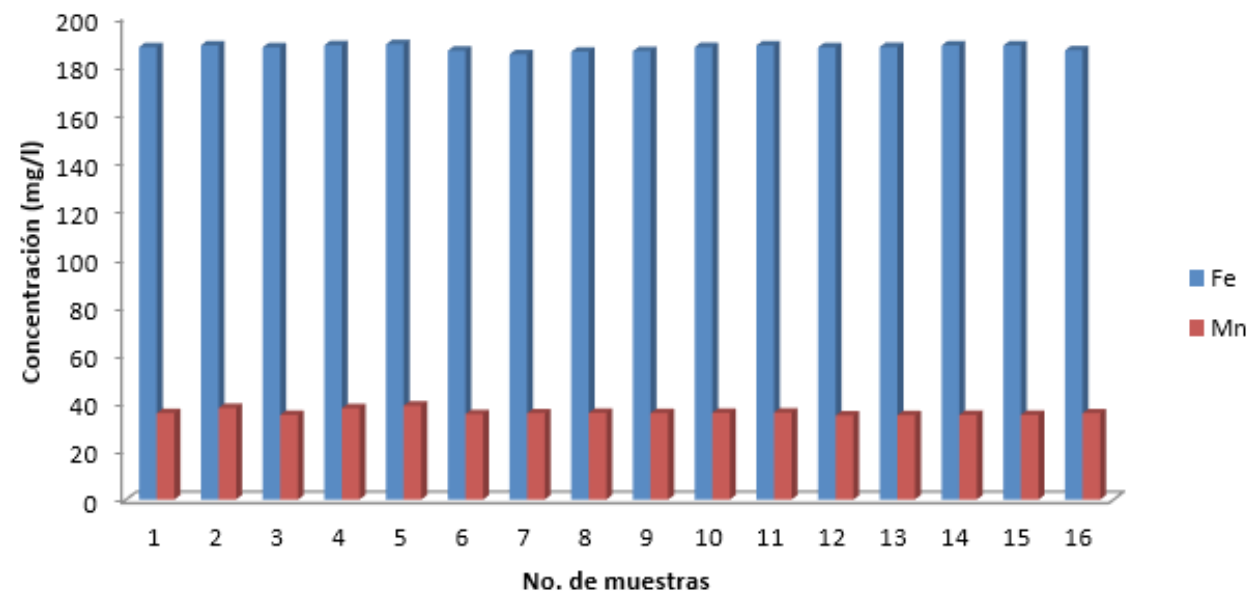


Figura 3.6. Parámetros microbiológicos. Eje x: Número de muestras y Eje y: Número más probable de microorganismos (NMP)

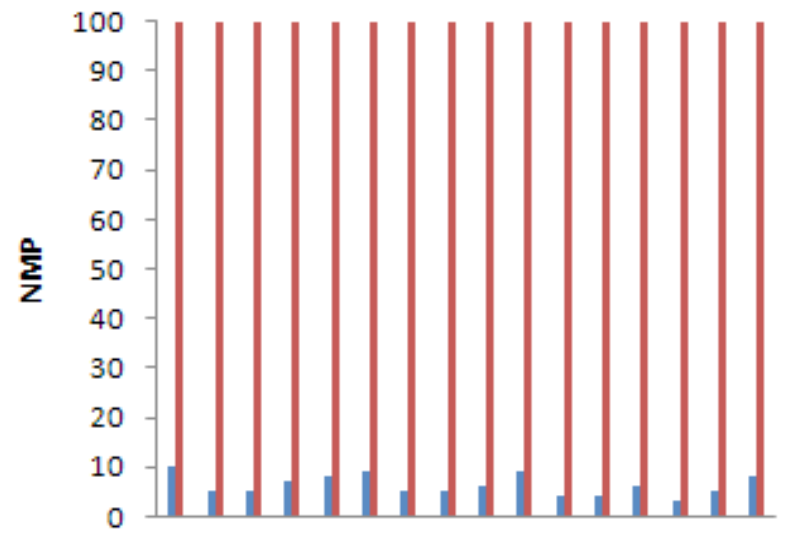

n Levaduras

- Hongos filamentosos

$\begin{array}{llllllllll}1 & 2 & 3 & 4 & 5 & 6 & 7 & 8 & 9 & 10111213141516\end{array}$

Número de muestras

Figura 3.7. Parámetros físico-químicos. Eje x: Número de muestras y Eje y: Concentración (mg/l)

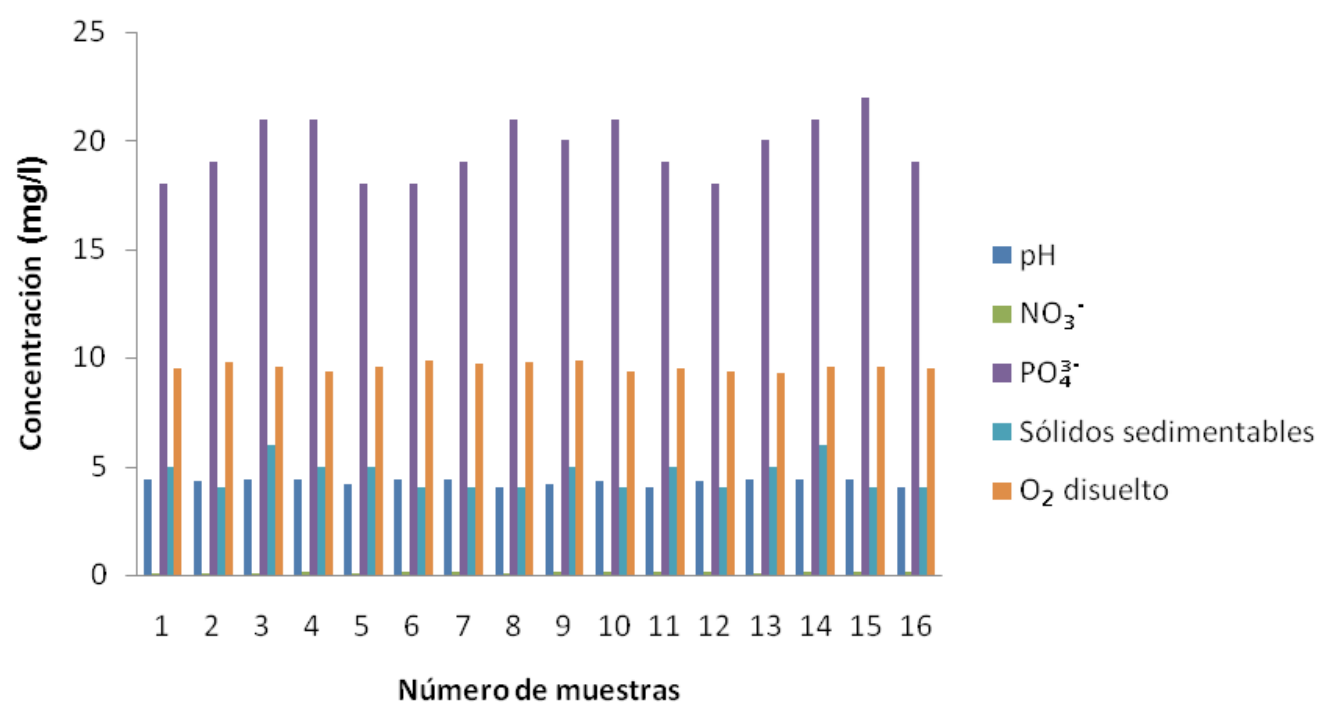

Desde el punto de vista microbiológico se puede apreciar que la proliferación de los hongos filamentosos se beneficia cuando se tratan compuestos ricos en azúcares y la reproducción de las levaduras también es favorecida cuando al mosto se le añade ácido sulfúrico y ácido clorhídrico. Este resultado se corresponde con los obtenidos por (Ajello, 2012) y (Madrigal, 2000), los que demostraron que las hifas de los hongos crecen con mayor rapidez cuando están en medios que contienen sacarosa, por lo que en la producción de etanol, a la salida de la columna de destilación (por donde sale el residual), es muy probable que continúe el crecimiento y la reproducción de levaduras.

La conductividad eléctrica se encuentra en el intervalo 13,99 mg/l-14,25 mg/l. De acuerdo a los resultados de los parámetros evaluados, en la Figura 3.7 se percibe que el $\mathrm{pH}$ de la vinaza de la destilería Héctor Molina es ácido, lo que concuerda con 
los estudios realizados por (Alfaro y Ocampo, 2012). Este también abordó temas sobre la conductividad eléctrica, infiriendo que este parámetro es alto en las vinazas debido a su poder de salinización.

Además de los parámetros antes mencionados, en la Figura 3.8 aparecen la DBO y la DQO, las cuales poseen altos valores, indicando que la vinaza es un residual con alto valor de carga orgánica, lo cual coincide con los resultados obtenidos por (Ávila,
2013), (Alfaro y Ocampo, 2012), (Gandini, 2013) y (Robles y Villalobos, 2011).

De igual forma se muestran en la Figura 3.9, la concentración del anión $\left(\mathrm{SO}_{4}^{2-}\right)$ y la concentración de los cationes $\left(\mathrm{Ca}^{2+}, \mathrm{Mg}^{2+}, \mathrm{Na}^{+}, \mathrm{K}^{+}\right.$), indicando que son altos debido al contenido de sales que hay en el medio de fermentación, lo cual se corrobora en las investigaciones de (Gandini, 2013) y (Robles y Villalobos, 2011).

Figura 3.8. Demanda de oxígeno. Eje de abscisas: Número de muestras y Eje de ordenadas: Concentración (mg/l)

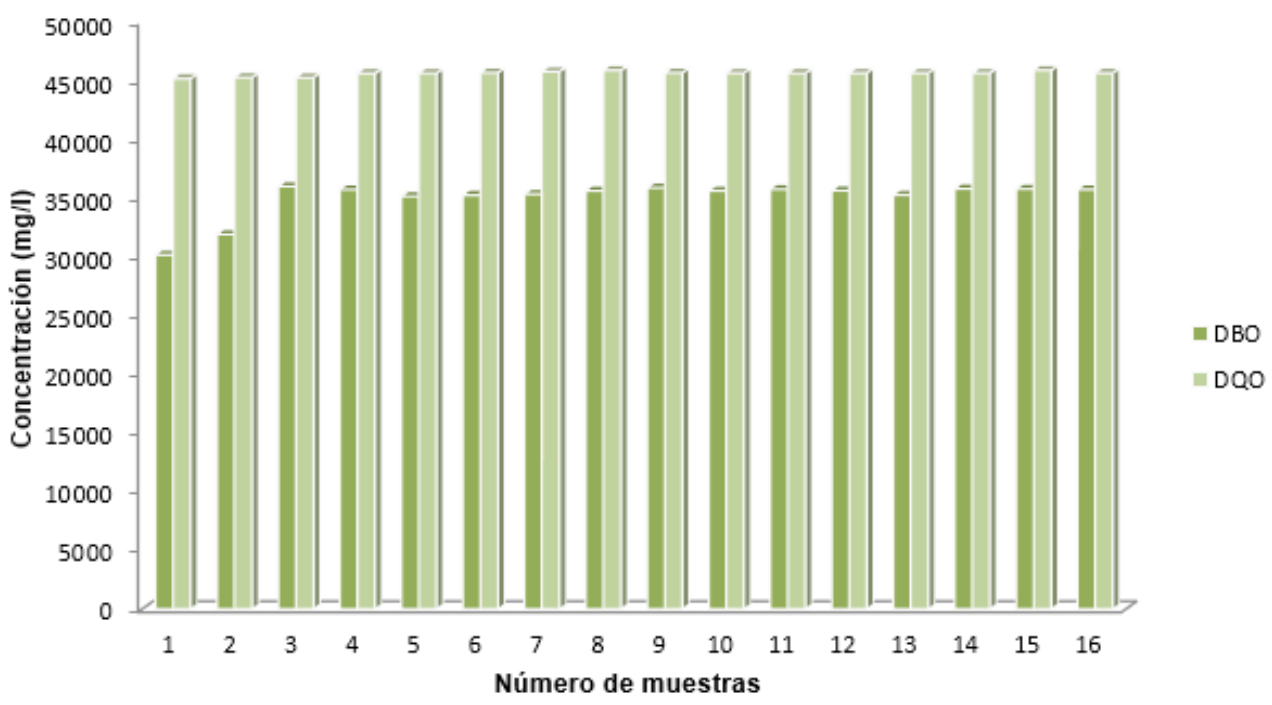

Figura 3.9. Parámetros químicos. Eje x: Número de muestras y Eje y: Concentración (mg/l)

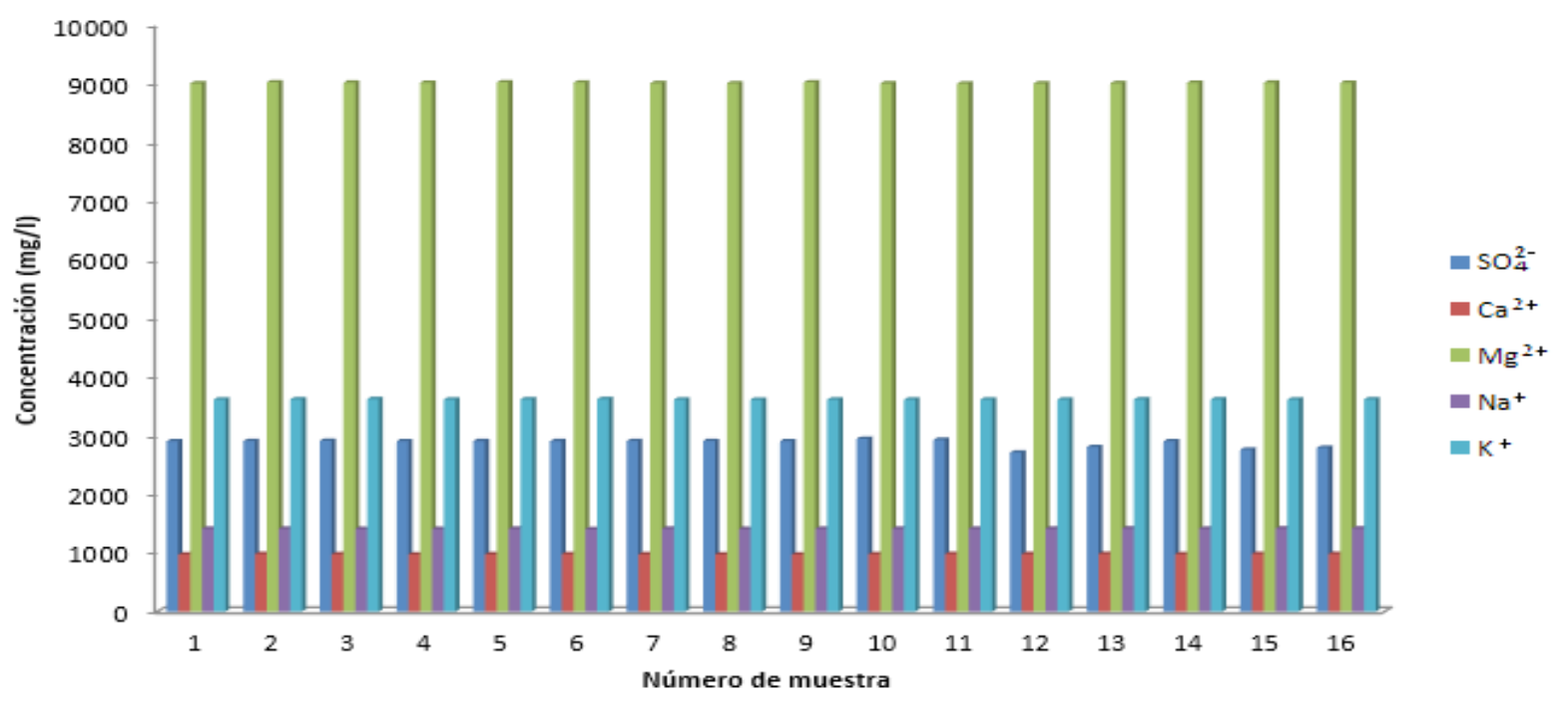




\section{Figura 3.10. Metales. Eje x: Número de muestras y Eje y: Concentración (mg/l)}

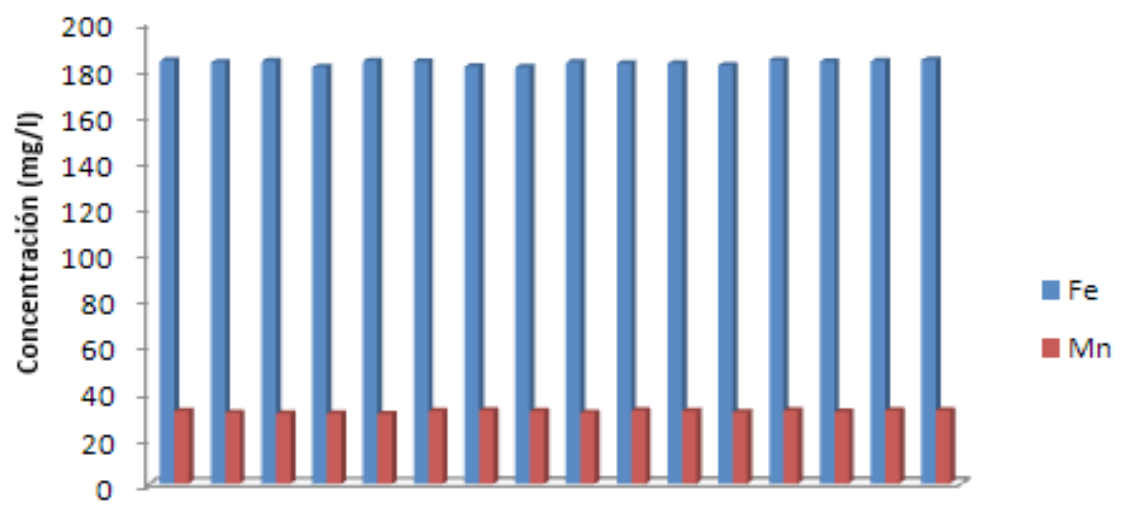

$\begin{array}{llllllllllllllll}1 & 2 & 3 & 4 & 5 & 6 & 7 & 8 & 9 & 10 & 11 & 12 & 13 & 14 & 15 & 16\end{array}$

No. de muestras

Los que poseen mayor concentración son: hierro y manganeso, ya que los restantes metales están presentes en concentraciones menores que 6 $\mathrm{mg} / \mathrm{l}$. Por consiguiente, en la vinaza hay presencia de metales pesados, lo que se corresponde con los estudios realizados por (Gandini, 2013) y (Robles y Villalobos, 2011).

\subsection{Comparación de las vinazas de ambas destillerías según su calidad}

A partir de los resultados de las Figuras 3.13.5, para los resultados experimentales determinados en la destilería San José, se calcularon las medias y las desviaciones estándar, para las variables que resultaron tener una distribución normal, así como las medianas y las desviaciones absolutas de las medianas (DAM) para las variables que resultaron tener una distribución no normal. Los resultados obtenidos se muestran en la Tabla 3.1 y en la Tabla 3.2.

TABLA 3.1. MEDIAS Y DESVIACIONES ESTÁNDAR DE LAS VARIABLES EN LA DESTILERÍA SAN JOSÉ

\begin{tabular}{c|c|c|c|} 
Variables & Unidad & Media & $\begin{array}{c}\text { Desviación } \\
\text { estándar }\end{array}$ \\
\hline Levaduras & UFC & 6,6 & 1,78 \\
\hline Temperatura & ${ }^{\circ} \mathrm{C}$ & 98,6 & 0,7 \\
\hline $\mathrm{pH}$ & - & 4,2 & 0,17
\end{tabular}

\begin{tabular}{|c|c|c|c|}
\hline $\begin{array}{l}\text { Conductividad } \\
\text { eléctrica }\end{array}$ & $\mathrm{mS} / \mathrm{cm}$ & 14,1 & 0,12 \\
\hline $\mathrm{SO}_{4}{ }^{2-}$ & $\mathrm{mg} / \mathrm{l}$ & $2.554,2$ & 56,5 \\
\hline $\mathrm{Ca}^{2+}$ & $\mathrm{mg} / \mathrm{l}$ & 954,2 & 4,5 \\
\hline $\mathrm{NO}_{3}^{-}$ & $\mathrm{mg} / \mathrm{l}$ & 0,1 & 0,01 \\
\hline $\mathrm{PO}_{4}^{3-}$ & $\mathrm{mg} / \mathrm{l}$ & 16,5 & 1,82 \\
\hline DBO & $\mathrm{mg} / \mathrm{l}$ & $28.130,6$ & $2.235,29$ \\
\hline DQO & $\mathrm{mg} / \mathrm{l}$ & $34.987,6$ & $2.346,25$ \\
\hline $\mathrm{O}_{2}$ disuelto & $\mathrm{mg} / \mathrm{l}$ & 9,6 & 0,1 \\
\hline $\mathrm{Ag}$ & $\mathrm{mg} / \mathrm{l}$ & 0,03 & 0,003 \\
\hline $\mathrm{Ba}$ & $\mathrm{mg} / \mathrm{l}$ & 2,2 & 0,04 \\
\hline $\mathrm{Cd}$ & $\mathrm{mg} / \mathrm{l}$ & 0,02 & 0,01 \\
\hline Co & $\mathrm{mg} / \mathrm{l}$ & 0,17 & 0,01 \\
\hline $\mathrm{Cu}$ & $\mathrm{mg} / \mathrm{l}$ & 5,00 & 0,02 \\
\hline $\mathrm{Fe}$ & $\mathrm{mg} / \mathrm{l}$ & 187,0 & 1,20 \\
\hline $\mathrm{Pb}$ & $\mathrm{mg} / \mathrm{l}$ & 0,05 & 0,03 \\
\hline $\mathrm{V}$ & $\mathrm{mg} / \mathrm{l}$ & 0,1 & 0,02 \\
\hline $\mathrm{Zn}$ & $\mathrm{mg} / \mathrm{l}$ & 4,7 & 0,47 \\
\hline $\begin{array}{c}\text { Sólidos } \\
\text { sedimentables }\end{array}$ & $\mathrm{ml} / \mathrm{l}$ & 2,7 & 0,93 \\
\hline
\end{tabular}

Tomando como base los resultados de las Figuras 3.6-3.10, se calcularon las medias y las desviaciones estándares, para las variables que resultaron poseer una distribución normal así como las medianas y las desviaciones absolutas de las 
medianas (DAM) para las variables que resultaron tener una distribución no normal de cada variable en la destilería Héctor Molina. Los resultados obtenidos se muestran en la Tabla 3.3 y en la Tabla 3.4 respectivamente.

TABLA 3.2. MEDIANAS Y DAM DE LAS VARIABLES EN LA DESTILERÍA SAN JOSÉ

\begin{tabular}{c|c|c|c|} 
Variables & Unidad & Mediana & DAM \\
\hline $\mathrm{Mg}^{2+}$ & $\mathrm{mg} / \mathrm{l}$ & $8.578,5$ & 11,0 \\
\hline $\mathrm{Na}$ & $\mathrm{mg} / \mathrm{l}$ & $1.370,5$ & 7,55 \\
\hline $\mathrm{K}^{+}$ & $\mathrm{mg} / \mathrm{l}$ & $3.576,0$ & 12,5 \\
\hline $\mathrm{Mn}$ & $\mathrm{mg} / \mathrm{l}$ & 35,7 & 0,5 \\
\hline $\mathrm{Ni}$ & $\mathrm{mg} / \mathrm{l}$ & 0,4 & 0,01 \\
\hline $\mathrm{Cr}$ & $\mathrm{mg} / \mathrm{l}$ & 0,195 & 0,005 \\
\hline
\end{tabular}

TABLA 3.3. MEDIAS Y DESVIACIONES ESTÁNDARES DE LAS VARIABLES EN LA DESTILERÍA HÉCTOR MOLINA

\begin{tabular}{|c|c|c|c|}
\hline Variables & Unidad & Media & $\begin{array}{c}\text { Desviación } \\
\text { estándar }\end{array}$ \\
\hline Levaduras & UFC & 6,1 & 2,0 \\
\hline Temperatura & ${ }^{\circ} \mathrm{C}$ & 98,8 & 0,71 \\
\hline $\mathrm{pH}$ & - & 4,2 & 0,15 \\
\hline $\begin{array}{c}\text { Conductividad } \\
\text { eléctrica }\end{array}$ & $\mathrm{mS} / \mathrm{cm}$ & 14,1 & 0,08 \\
\hline $\mathrm{Mg}^{2+}$ & $\mathrm{mg} / \mathrm{l}$ & $9.008,1$ & 6,71 \\
\hline $\mathrm{K}^{+}$ & $\mathrm{mg} / \mathrm{l}$ & $3.613,1$ & 2,73 \\
\hline $\mathrm{NO}_{3}^{-}$ & $\mathrm{mg} / \mathrm{l}$ & 0,1 & 0,01 \\
\hline $\mathrm{PO}_{4}^{3-}$ & $\mathrm{mg} / \mathrm{l}$ & 19,6 & 1,35 \\
\hline DQO & $\mathrm{mg} / \mathrm{l}$ & $45.597,8$ & 202,301 \\
\hline $\mathrm{O}_{2}$ disuelto & $\mathrm{mg} / \mathrm{l}$ & 9,5 & 0,18 \\
\hline $\mathrm{Ag}$ & $\mathrm{mg} / \mathrm{l}$ & 0,01 & 0,01 \\
\hline $\mathrm{Ba}$ & $\mathrm{mg} / \mathrm{l}$ & 3,0 & 0,01 \\
\hline $\mathrm{Cd}$ & $\mathrm{mg} / \mathrm{l}$ & 0,03 & 0,01 \\
\hline Co & $\mathrm{mg} / \mathrm{l}$ & 0,2 & 0,01 \\
\hline $\mathrm{Cu}$ & $\mathrm{mg} / \mathrm{l}$ & 4,6 & 0,01 \\
\hline $\mathrm{Fe}$ & $\mathrm{mg} / \mathrm{l}$ & 182,8 & 1,04 \\
\hline $\mathrm{Mn}$ & $\mathrm{mg} / \mathrm{l}$ & 31,2 & 0,56 \\
\hline $\mathrm{Ni}$ & $\mathrm{mg} / \mathrm{l}$ & 0,5 & 0,01 \\
\hline $\mathrm{Pb}$ & $\mathrm{mg} / \mathrm{l}$ & 0,1 & 0,01 \\
\hline $\mathrm{Zn}$ & $\mathrm{mg} / \mathrm{l}$ & 4,7 & 0,06 \\
\hline $\begin{array}{c}\text { Sólidos } \\
\text { sedimentables }\end{array}$ & $\mathrm{ml} / \mathrm{l}$ & 4,6 & 0,71 \\
\hline
\end{tabular}

TABLA 3.4. MEDIANAS Y DAM DE LAS VARIABLES DE LA DESTILERÍA HÉCTOR MOLINA

\begin{tabular}{c|c|c|c} 
Variables & Unidad & Mediana & DAM \\
\hline $\mathrm{SO}_{4}^{2-}$ & $\mathrm{mg} / \mathrm{l}$ & $2.902,0$ & 3,0 \\
\hline $\mathrm{Ca}^{2+}$ & $\mathrm{mg} / \mathrm{l}$ & 976,0 & 3,0 \\
\hline $\mathrm{DBO}$ & $\mathrm{mg} / \mathrm{l}$ & $35.619,0$ & 207,5
\end{tabular}

Con los valores reportados en las Tablas 3.1-3.4 se desarrollaron las pruebas para comparar si existían diferencias significativas entre las vinazas de las dos destilerías. En la Tabla 3.5 aparecen los resultados estadísticos correspondientes a las pruebas de hipótesis. Para comparar las medias se utilizó la prueba t y para comparar las desviaciones estándares se aplicó la prueba F. Del mismo modo se muestra en la Tabla 3.6 los resultados estadísticos que arrojó la prueba W de Mann-Whitney (Wilcoxon) para la comparación de las medianas. Estas pruebas se realizaron para un nivel de confianza de 95\%. Para valores de P mayores o iguales que 0,05 no hay diferencias estadísticamente significativas entre las muestras. En la Tabla $\mathbf{3 . 5}$ se destacan en cursiva las variables para las que existen diferencias estadísticamente significativas.

TABLA 3.5. DIFERENCIAS SIGNIFICATIVAS DE LAS VARIABLES SEGÚN DISTRIBUCIÓN NORMAL

\begin{tabular}{c|c|c|c|c}
\multirow{2}{*}{ Variables } & \multicolumn{2}{|c|}{ Prueba t } & \multicolumn{2}{c}{ Prueba F } \\
\cline { 2 - 5 } & $\mathbf{t}$ & valor-P & $\mathbf{F}$ & valor-P \\
\hline Temperatura & $-1,0931$ & 0,2830 & 0,9701 & 0,9540 \\
\hline $\mathrm{pH}$ & $-0,7202$ & 0,4769 & 1,3851 & 0,5357 \\
\hline $\begin{array}{c}\text { Conductividad } \\
\text { eléctrica }\end{array}$ & $-0,2020$ & 0,8412 & 2,4039 & 0,0999 \\
\hline $\mathrm{NO}_{3}{ }^{1-}$ & 4,1084 & 0,0003 & 0,4421 & 0,1250 \\
\hline $\mathrm{PO}_{4}{ }^{--}$ & $-5,6115$ & 0,0000 & 1,8223 & 0,2565 \\
\hline $\mathrm{DQO}$ & 17,9043 & 0,0000 & 0,007 & 0,0000 \\
\hline $\begin{array}{c}\text { Sólidos } \\
\text { sedimentables }\end{array}$ & $-6,3767$ & $4,8 E-7$ & 1,6774 & 0,3271 \\
\hline $\mathrm{O}_{2}$ disuelto & 0,9568 & 0,3463 & 0,6282 & 0,3780 \\
\hline $\mathrm{Ag}$ & 14,7169 & 0,0000 & 0,5973 & 0,3290 \\
\hline $\mathrm{Ba}$ & $-71,6425$ & 0,0000 & 31,5278 & $2,5 E-8$ \\
\hline $\mathrm{Cd}$ & $-1,5719$ & 0,12640 & 2,2378 & 0,1299 \\
\hline $\mathrm{Co}$ & $-1,5719$ & 0,12640 & 2,2378 & 0,1299 \\
\hline $\mathrm{Cu}$ & 53,9812 & 0,0000 & 3,6477 & 0,0169 \\
\hline $\mathrm{Fe}$ & 10,5602 & $1,3 E-11$ & 1,3268 & 0,5907 \\
\hline Levaduras & 0,7457 & 0,4616 & 0,6741 & 0,4540 \\
\hline & & & &
\end{tabular}


De la tabla se aprecia que, al comparar las medias, no existen diferencias significativas entre las variables temperatura, $\mathrm{pH}$, conductividad eléctrica, oxígeno disuelto, contenidos de $\mathrm{Cd}$, Co y levaduras. Por otra parte, los contenidos de $\mathrm{NO}_{3}{ }^{-}, \mathrm{PO}_{4}{ }^{3-}, \mathrm{DQO}$, sólidos sedimentables, $\mathrm{Ag}, \mathrm{Ba}, \mathrm{Cu}$ y Fe sí presentan diferencias estadísticamente significativas.

TABLA 3.6. DIFERENCIAS SIGNIFICATIVAS DE LAS VARIABLES SEGÚN DISTRIBUCIÓN NO NORMAL

\begin{tabular}{c|c|c|}
\multirow{2}{*}{ Variables } & \multicolumn{2}{|c|}{$\begin{array}{c}\text { Prueba W de Mann-Whitney } \\
\text { (Wilcoxon) }\end{array}$} \\
\cline { 2 - 3 } & $\mathbf{W}$ & valor-P \\
\hline $\mathrm{Mg}^{2+}$ & 128,0 & $1,5 E-6$ \\
\hline $\mathrm{Na}^{+}$ & 128,0 & $1,4 E-6$ \\
\hline $\mathrm{K}^{+}$ & 128,0 & $1,4 E-6$ \\
\hline $\mathrm{Mn}$ & $-128,0$ & $1,5 E-6$ \\
\hline $\mathrm{Ni}$ & 128,0 & $1,3 E-6$ \\
\hline $\mathrm{Cr}$ & 102,0 & $1,0 E-4$ \\
\hline $\mathrm{SO}_{4}^{2-}$ & 128,0 & $1,5 E-6$ \\
\hline$D B O$ & 125,0 & $2,7 E-6$ \\
\hline $\mathrm{Ca}^{2+}$ & 128,0 & $1,4 E-6$ \\
\hline
\end{tabular}

De la prueba de comparación de medianas se aprecia que los contenidos de $\mathrm{Mg}^{2+}, \mathrm{Na}^{+}, \mathrm{K}^{+}, \mathrm{Mn}, \mathrm{Ni}$,
$\mathrm{Cr}, \mathrm{SO}_{4}{ }_{4}^{2-}, \mathrm{Ca}^{2+}$ y DBO presentan diferencias estadísticamente significativas.

A partir del análisis estadístico realizado para la comparación de las vinazas entre las destilerías San José y Héctor Molina se puede observar que existen diferencias significativas como se muestra en las Tablas 3.5 y 3.6. En resumen, de 24 parámetros que caracterizan estas vinazas, son estadísticamente diferentes 17 , lo que puede estar asociado a la diferencia entre los sistemas de fermentación con y sin enfriamiento, las cepas usadas, la naturaleza del ácido utilizado para regular $\mathrm{pH}$ y el sistema de destilación. Por tanto, puede afirmarse que, en general, difieren entre sí las vinazas de ambas destilerías.

Según el estudio de correlación realizado entre las variables $\mathrm{DBO}, \mathrm{pH}$, temperatura y $\mathrm{O}_{2}$ disuelto de las vinazas de ambas destilerías, se pudo determinar que en ambos casos existe una correlación positiva significativa para un $95 \%$ de probabilidad entre las variables DBO y temperatura, como se muestra en las Tablas 3.7 y 3.8 .

Las otras variables no mostraron correlación según los datos analizados.

TABLA 3.7. CORRELACIONES OBTENIDAS ENTRE LAS VARIABLES DBO, DQO, PH, TEMPERATURA Y O ${ }_{2}$ DISUELTO DE LA VINAZA DE LA DESTILERÍA DE SAN JOSÉ. LAS CORRELACIONES QUE TIENEN SIMPLE SUBRAYADO SON SIGNIFICATIVAS PARA $P<0,05$

\begin{tabular}{c|c|c|c|c|c} 
& DBO & DQO & $\mathrm{O}_{2}$ disuelto & $\mathrm{pH}$ & Temperatura \\
\hline DBO & & 0,2947 & $-0,1619$ & $-0,1851$ & $-0,1658$ \\
\hline & & $\underline{0,0079}$ & 0,5492 & 0,4925 & $\underline{0,0095}$ \\
\hline DQO & 0,2947 & & 0,1172 & $-0,4061$ & $-0,6551$ \\
\hline $\mathrm{O}_{2}$ disuelto & $\underline{0,0079}$ & & 0,6654 & 0,1186 & 0,0559 \\
\hline & $-0,1619$ & 0,1172 & & 0,0193 & 0,1551 \\
\hline pH & 0,5492 & 0,6654 & & 0,9434 & 0,5662 \\
\hline & $-0,1851$ & $-0,4061$ & 0,0193 & & 0,1998 \\
\hline Temperatura & 0,4925 & 0,1186 & 0,9434 & & 0,4582 \\
\hline & $-0,1658$ & $-0,6551$ & 0,1551 & 0,1998 & \\
\hline & 0,0095 & 0,0559 & 0,5662 & 0,4582 & \\
\hline
\end{tabular}


TABLA 3.8. CORRELACIONES OBTENIDAS ENTRE LAS VARIABLES DBO, DQO, PH, TEMPERATURA Y O DISUELTO DE LA VINAZA DE LA DESTILERÍA HÉCTOR MOLINA. LAS CORRELACIONES QUE TIENEN SIMPLE SUBRAYADO SON SIGNIFICATIVAS PARA $P<0,05$

\begin{tabular}{c|c|c|c|c|c} 
& DBO & DQO & $\mathrm{O}_{2}$ disuelto & $\mathrm{pH}$ & Temperatura \\
\hline DBO & & 0,6937 & $-0,0586$ & $-0,2163$ & 0,5244 \\
\hline & & $\underline{0,0229}$ & 0,8294 & 0,4211 & 0,0371 \\
\hline DQO & 0,6937 & & 0,1062 & $-0,2720$ & 0,4957 \\
\hline & 0,0229 & & 0,6955 & 0,3081 & 0,0508 \\
\hline $\mathrm{O}_{2}$ disuelto & $-0,0586$ & 0,1062 & & $-0,0595$ & $-0,4100$ \\
\hline & 0,8294 & 0,6955 & & 0,8268 & 0,1147 \\
\hline pH & $-0,2163$ & $-0,2720$ & $-0,0595$ & & $-0,3464$ \\
\hline & 0,4211 & 0,3081 & 0,8268 & & 0,1887 \\
\hline Temperatura & 0,5244 & 0,4957 & $-0,4100$ & $-0,3464$ & \\
\hline & 0,0371 & 0,0508 & 0,1147 & 0,1887 & \\
\hline
\end{tabular}

TABLA 3.9. COMPARACIÓN DE LAS VINAZAS DE LAS DESTILERÍAS CON LA NC 27:2012

\begin{tabular}{c|c|c|c|c|c}
\multirow{2}{*}{ Variables } & \multirow{2}{*}{ Unidad } & San José & \multicolumn{2}{|c|}{ Héctor Molina } & \multirow{2}{*}{$\begin{array}{c}\text { Norma establecida en } \\
\text { NC 27:2012 }\end{array}$} \\
\cline { 3 - 5 } & & Media & Media & Mediana & \\
\hline $\mathrm{pH}$ & - & 4,2 & 4,2 & & $6-9$ \\
\hline Temperatura & ${ }^{\circ} \mathrm{C}$ & 98,6 & 98,8 & & $<50$ \\
\hline Conductividad eléctrica & $\mu \mathrm{S} / \mathrm{cm}$ & 14.100 & 14.100 & & $<4.000$ \\
\hline Sólidos sedimentables & $\mathrm{ml} / \mathrm{l}$ & 2,7 & 4,6 & & $<10$ \\
\hline $\mathrm{DBO}_{5}{ }^{20}$ & $\mathrm{mg} / \mathrm{l}$ & $28.130,6$ & & $35.619,0$ & $<300$ \\
\hline $\mathrm{DQO}$ & $\mathrm{mg} / \mathrm{l}$ & $34.987,6$ & $45.597,8$ & & $<700$ \\
\hline $\mathrm{Cd}$ & $\mathrm{mg} / \mathrm{l}$ & 0,02 & 0,03 & & $<0,30$ \\
\hline $\mathrm{Cu}$ & $\mathrm{mg} / \mathrm{l}$ & 5,00 & 4,60 & & $<5,00$ \\
\hline $\mathrm{Pb}$ & $\mathrm{mg} / \mathrm{l}$ & 0,05 & 0,12 & & $<1,00$ \\
\hline $\mathrm{Zn}$ & $\mathrm{mg} / \mathrm{l}$ & 4,7 & 4,7 & & $<5,00$ \\
\hline
\end{tabular}

Esta correlación positiva entre las variables DBO y temperatura se explica debido que al aumentar la temperatura aumenta la DBO, lo cual ha sido planteado por (Suárez, et al. 2013) y responde al incremento de los procesos fisiológicos de los microorganismos en un medio líquido cuando aumenta la temperatura, la que afecta la vida acuática, las reacciones químicas y sus velocidades. Además existe correlación positiva entre DBO y DQO, lo cual se corresponde por los estudios realizados por (Aguirre 2013), los mismos expresan que la DQO engloba la DBO y mantienen su relación para cada tipo de residual.

Sin embargo, los valores de correlación de ambas destilerías, sí muestran una pequeña diferencia ya que como se puede observar en las Tablas $3.7 \mathrm{y}$ 3.8, la destilería San José tiene un valor de correlación de 0,0095 y la destilería Héctor Molina tiene un valor de correlación de 0,0371, para un 95\% de probabilidad, lo que indica que la destilería Héctor Molina posee una mayor correlación entre DBO y 
temperatura que la destilería San José. Esto se evidencia en los valores de $\mathrm{DBO}_{5}{ }^{20}$ pues experimentan mayor variabilidad con respecto a los valores de temperatura. Igualmente se pudo comprobar que la destilería Héctor Molina tiene un valor de correlación entre las variables $\mathrm{DQO}$ y $\mathrm{DBO}_{5}{ }^{20}$ mayor que la destilería San José.

A partir de la media o mediana de cada variable de las vinazas de ambas destilerías se realizó una comparación de los resultados con la norma establecida NC 27:2012 como se muestra en la Tabla 3.9.

Como se evidencia en la Tabla 3.9, hay parámetros que no cumplen con la norma establecida: $\mathrm{pH}$, temperatura, conductividad eléctrica, $\mathrm{DBO}_{5}{ }^{20}$ y DQO.

Es por ello que es imprescindible diseñar un sistema de tratamiento de estos, no solo para cumplir la norma sino para reducir la contaminación que provocan al medio ambiente, fundamentalmente en lo relativo a la contaminación del suelo y las aguas subterráneas, recursos naturales indispensables para la vida en el planeta. Dada la relación obtenida entre $\mathrm{DBO}_{5}{ }^{20}$ y DQO en la caracterización de las vinazas, se recomienda la valoración de un sistema de tratamiento biológico anaerobio seguido de algún post-tratamiento como la oxidación avanzada, que pudiera ser similar para ambas. No obstante, esto debe ser evaluado previamente.

\section{CONCLUSIONES}

Las vinazas de las destilerías Héctor Molina y San José mostraron comportamiento similares en cuanto a pH, DBO y DQO. El pH de las mismas se comportó ácido, los valores de DBO y DQO son altos, evidenciando que en este residual son variadas las sustancias biodegradables y las no biodegradables. Con respecto a la microbiología se destaca la proliferación de hongos filamentosos y la presencia de levaduras.

Se realizó la comparación estadística entre las variables de las vinazas de las dos destilerías. Esta comparación arrojó que existen diferencias significativas entre ellas para un 95\% de confianza en $\mathrm{NO}_{3}^{-}$ , $\mathrm{PO}_{4}{ }^{3-}, \mathrm{SO}_{4}{ }^{2-}, \mathrm{Mg}^{2+}, \mathrm{Na}^{+}, \mathrm{K}^{+}, \mathrm{DQO}, \mathrm{DBO}, \mathrm{Ag}, \mathrm{Ba}, \mathrm{Fe}, \mathrm{Mn}$,
Ni, Cr, Ca y sólidos sedimentables. Además se compararon con la legislación vigente (NC 27/2012) encontrándose que las variables: DBO, DQO, conductividad eléctrica, $\mathrm{pH}$ y temperatura no cumplen con los límites máximos permisibles de esta norma.

Lo anterior demuestra la necesidad de establecer un sistema que reduzca el nivel de vinazas, así como el tratamiento de las vinazas antes de su emisión.

\section{REFERENCIAS}

Aguirre, M. (2013). “¿Qué diferencia hay entre DBO y DQO?", visitado en octubre de 2014, from www. aguaydepuración.com.

Ajello, G. (2012). CDC Laboratory Manual for medical Mycology. Revista paraguaya de microbiología. 1-7.

Alfaro Portuguez, R. and R. Ocampo Chinchilla (2012). Cambios físico-químicos provocados por la vinaza en un suelo vertisol en Costa Rica., Departamento de Investigación y Extensión de la Caña de Azúcar (DIECA- LAICA Costa Rica).

Ávila Sánchez, D. (2013). Recuperación por adsorción del potasio en vinaza cruda mediante el empleo de zeolita tipo clinoptilotita. Ingeniera Química Memoria descriptiva presentada en opción al título de máster en Ingeniería Ambiental, Instituto Superior Politécnico José Antonio Echeverría.

Díaz Betancourt, R. (1987). Tratamiento de agua y aguas residuales. Imprenta Andre Voisin.

Diez Torres, F. (mayo, 2008). Alternativas de tratamiento de residuales de producción alcoholera. Presentación para la empresa Alur de Uruguay. Uruguay.

Gandini Ayerbe, M. A. (2013). “Caracterización ambiental de las vinazas de residuos de caña de azúcar resultantes de la producción de etanol.".

Lezcano, P. and L. Mora (2011). Las vinazas de destilerías de alcohol. Contaminación ambiental o tratamiento para evitarlo. VIII Encuentro de Nutrición y producción de Animales Monogástricos. Instituto de Ciencia Animal. San José de las Lajas, La Habana, Cuba.

Madrigal Pulido, J. A. (2000). Evaluación de las vinazas tequileras como sustratos para el cultivo en estado sólido y líquido de hongos filamentosos. Tesis profesional para obtener el título de Licenciado en Biología., Universidad de Guadalajara. 
Normalización, Cómite Técnico (2012). Norma cubana. Vertimiento de aguas residuales a las aguas terrestres y al alcantarrillado. Específicaciones. La Habana. Cuba.

Perdigón Martín, S. M., J. Obregón Luna, et al. (2005). Impacto sobre el medio ambiente de las vinazas de los jugos de caña energética más miel final en la destilería Paraíso. Tesis presentada en opción del grado de Master en Gestión Ambiental y Protección de los Recursos Naturales, Universidad de Matanzas.

Robles Gonzáles, V. and F. Villalobos Castillejos (2011). "Vinazas Mezcaleras: Un problema de contaminación ambiental."

Suárez Alvarez, G., R. Valdés Herrera, et al. (2013). Efecto de la temperatura y el período de oxidación sobre la demanda bioquímica de oxígeno de aguas residuales pesqueras. IV Taller Internacional Contaminación y Protección del Medio Ambiente. La Habana., CONyMA.

\section{PARA CITAR ESTE ARTÍCULO TO REFERENCE THIS ARTICLE / PARA CITAR ESTE ARTIGO /}

Pérez Oliva, Y.; Pérez Ones, O.; Zumalacárregui de Cárdenas, L. (2017). Caracterización química, física y microbiológica de dos vinazas cubanas. Revista EIA, 14(28), julio-diciembre, pp. 29-43. [Online]. Disponible en: https://doi.org/10.24050/ reia.v14i28.695 